\title{
Determinantes do grau de evidenciação de risco de crédito pelos bancos brasileiros
}

\author{
Determinants of the credit risk disclosure level by Brazilian banks
}

\author{
José Alves Dantas \\ Professor do Centro Universitário Unieuro e Doutorando do Programa de Pós-Graduação \\ Multiinstitucional e Inter-Regional de Ciências Contábeis - UnB/UFPB/UFRN \\ E-mail: alves.dantas@bcb.gov.br
}

\section{Fernanda Fernandes Rodrigues}

Professora da Universidade de Brasília e Doutoranda do Programa de Pós-Graduação

Multiinstitucional e Inter-Regional de Ciências Contábeis - UnB/UFPB/UFRN

E-mail: fernandes@unb.br

\section{Jomar Miranda Rodrigues}

Professor da Universidade de Brasília e Doutorando do Programa de Pós-Graduação

Multiinstitucional e Inter-Regional de Ciências Contábeis - UnB/UFPB/UFRN

E-mail: jomar@unb.br

\section{Lucio Rodrigues Capelletto}

Professor Pesquisador Associado do Programa de Pós-Graduação Multiinstitucional e InterRegional de Ciências Contábeis - UnB/UFPB/UFRN

E-mail: capelletto@bcb.gov.br

Recebido em 28.10.2009 * Aceito em 02.02.2010 * $2^{\mathrm{a}}$ versão aceita em 08.03.2010

\section{RESUMO}

Estudos que avaliam o nível de evidenciação praticado pelas organizações têm adquirido relevância na literatura contábil, na medida em que a evidenciação assume papel cada vez mais importante para a redução da assimetria de informação entre os diversos agentes econômicos. No caso do sistema financeiro, considerando suas peculiaridades, a transparência é essencial para garantir a confiabilidade e a estabilidade do sistema. O presente estudo teve por objetivo central avaliar o grau de transparência do risco de crédito praticado pelas instituições financeiras brasileiras, tendo por referência as divulgações qualitativas e quantitativas requeridas pelo Acordo de Basiléia II. Com base nas Informações Financeiras Trimestrais dos 50 maiores bancos, os resultados das análises revelaram, inicialmente, que o grau de evidenciação apresentou crescimento paulatino e constante no período, mas ainda atende a menos de $40 \%$ dos itens requeridos. Por meio de regressão, com o uso de dados em painel, foi constatado que o grau de evidenciação do risco de crédito de um período é explicado, inicialmente, pela divulgação do período anterior. Também foi constatado que o nível de evidenciação é explicado pela vigência de Basiléia II, pelo porte das instituições, pelo nível de rentabilidade, pelo índice de imobilização e pela listagem das ações na Bovespa.

Palavras-chave: Risco de Crédito. Basiléia II. Bancos. Evidenciação. Transparência. 


\section{ABSTRACT}

Studies assessing organizations' level of disclosure have gained relevance in Accounting literature, as disclosure progressively assumes a more important role in the reduction of information asymmetry amongst various economic agents. As for the peculiarities of the Financial System, transparency is essential to achieve reliability and stability. The central aim of this study is to assess the degree of credit risk disclosure implemented by Brazilian financial institutions, benchmarked against the qualitative and quantitative disclosures required by the Basel II Accord. Based on the analysis of quarterly financial data of the fifty biggest banks, the results of the research show, initially, that the level of disclosure presented a slow and steady growth in the period, despite complying with less than forty percent of the information items required. The Study's Panel Data Regression Analysis revealed that the degree of credit risk disclosure of a period is initially explained by the disclosure of the previous period. It also indicated that the level of disclosure is explained by the Basel II Accord's effectiveness, the size of the organization, the level of returns, the fixed-asset to equity-capital ratio and also by the shares listed on Bovespa.

Keywords: Credit Risk. Basel II. Banks. Disclosure. Transparency.

\section{INTRODUÇAO}

Por meio do documento Convergência Internacional de Mensuração de Capital e Padrões de Capital - Estrutura Revisada, que ficou conhecido como Acordo de Basiléia II, o Comitê de Supervisão Bancária de Basiléia definiu uma estrutura com o objetivo de fortalecer a solidez e a estabilidade do sistema bancário internacional e manter consistência suficiente para que o regulamento de adequação de capital não seja fonte significativa de desigualdade competitiva entre os bancos internacionalmente ativos (BCBS, 2004).

Além de apresentar alternativas mais sofisticadas para o cálculo do capital mínimo regulamentar, buscando aproximá-lo do capital econômico calculado pelos próprios bancos na gestão dos seus negócios, uma das principais inovações do Acordo foi estabelecer os requisitos de transparência para as instituições financeiras, constituindo-se em elemento essencial para a redução dos riscos inerentes ao sistema financeiro.

Entre esses riscos, o relevante é o risco de crédito (BCBS, 2000), definido como a possibilidade de um tomador de empréstimo ou contraparte não cumprir suas obrigações conforme os termos acordados, que está relacionado à essência das atividades de uma instituição financeira. Assim, a adequada evidenciação do risco de crédito de uma instituição financeira é determinante apara a correta avaliação de sua situação econômico-financeira.

Cabe ressaltar que, embora o Comitê de Basiléia não possua autoridade supranacional formal e seus pronunciamentos não tenham força legal (BCBS, 2009), o Banco Central do Brasil (BCB) comunicou a intenção de implementar a nova estrutura de capital estabelecida 
por Basiléia II (BCB, 2004 e 2007), com observância às condições, às peculiaridades e do estágio de desenvolvimento do mercado brasileiro. O estabelecimento de um cronograma para a edição das normas necessárias à criação do arcabouço legal necessário à implementação das recomendações relacionadas aos requerimentos de capital ratificou a intenção de adotar as diretrizes de Basiléia II no Sistema Financeiro Nacional (SFN), com destaque para os processos de supervisão e de transparência e disciplina de mercado.

Não obstante o consenso sobre os benefícios da maior transparência, há contumaz resistência nas organizações quanto ao aumento do grau de evidenciação, conforme Dantas et al. (2005). Algumas consideram que a maior evidenciação disponibiliza informações à concorrência, expõe estratégias de negócios e provoca a perda de competitividade.

Ante o exposto, este estudo tem dois objetivos centrais: (i) avaliar se houve impacto na forma como os bancos divulgam suas informações sobre risco de crédito a partir da divulgação dos parâmetros de Basiléia II, tendo em vista que, embora sua implementação no Brasil ainda esteja em processo, o $\mathrm{BCB}$ comunicou que seguirá os requerimentos de transparência recomendados no Acordo e (ii) identificar quais são as variáveis determinantes do nível de divulgação de risco de crédito das instituições financeiras no Brasil.

Para o alcance desses objetivos, serão examinadas as informações divulgadas sobre o risco de crédito no documento Informações Financeiras Trimestrais (IFT), referentes ao período de 2001 a 2008, das instituições e conglomerados financeiros que integram o relatório 50 Maiores Bancos no SFN, na posição de dezembro de 2008. O exame verificará o atendimento às exigências previstas no Pilar 3 de Basiléia II para a divulgação de informações relativas ao risco de crédito.

A análise das $\mathrm{IFT}^{1}$ é justificada pelo fato de proporcionar, ao público interessado, acesso tempestivo a informações confiáveis e diversificadas, contribuindo para o fortalecimento do SFN, ao disponibilizar um conjunto de informações mais amplo que os exigidos normativamente das demonstrações contábeis publicadas pelas instituições financeiras (FERREIRA; ARAÚJO, 2004).

\footnotetext{
${ }^{1}$ Instituídas pelo BCB, por meio da Circular 2.990, de 28.6.2000. 


\section{REVISÃO DE LITERATURA}

\subsection{Teoria da Divulgação}

Segundo Verrecchia (2001), a teoria da divulgação está fundamentada em alguns pressupostos que influenciam a precificação dos ativos, tais como: i) os investidores são diversamente informados; ii) os investidores fazem inferências racionais dos preços de mercado; iii) os investidores antecipam racionalmente a divulgação; iv) os investidores são informados com qualidade diversa e heterogênea; v) os investidores interpretam a divulgação de maneira diferenciada; vi) os investidores agregam opiniões diversas, ou seja, alguns agentes incorporam a divulgação nas suas expectativas posteriores e vii) os investidores condicionam as suas opiniões de acordo com estímulos econômicos diversos, ou seja, eles fazem inferências racionais tanto para preços de mercado quanto para volume de transações.

O alcance da teoria da divulgação é mais facilmente compreendido ao serem consideradas as três dimensões em que está baseado, conforme Verrecchia (2001): associação, julgamento e eficiência. Baseia-se em associação, na medida em que investiga a relação dos usuários das informações e as decisões por eles tomadas. Em julgamento, quando procura identificar como os usuários tomam decisões e as empresas decidem divulgar ou não determinada informação. Por último, está baseada em eficiência ao verificar quais configurações de informações de divulgação são preferidas.

Coerente com essa abrangência, Iudícibus (2000) associa a divulgação ou disclosure diretamente ao objetivo da Contabilidade, que é prover informação útil à tomada de decisões econômicas, embora uma conclusão sobre o que é útil ou não ao processo decisório do usuário não seja simples de avaliar. Isso porque cada usuário requer um tipo de informação que atenda às suas necessidades para a tomada de decisão e a Contabilidade ainda não dispõe de meios capazes de atender, igualmente, bem as necessidades específicas de cada um. Não obstante essa dificuldade, os usuários devem ser atendidos com informações claras e compreensíveis a fim de poderem avaliar a posição econômico-financeira da empresa e obterem conclusões que contribuam para formar seu juízo de forma confiável (NIYAMA, 1989).

Se a transparência das informações é importante para a tomada de decisões econômicas, como mencionado, no sistema financeiro é imprescindível, pois somente exerce atividade de intermediação se houver a confiança das pessoas nas instituições integrantes do sistema. Nesse particular, a transparência por parte das instituições financeiras é entendida como a divulgação pública de informação segura e tempestiva que possibilite ao usuário 
avaliar a condição financeira, o desempenho nos negócios, o perfil de risco e as práticas de gerenciamento de risco (BCBS, 1998).

Aliás, foi a falta de confiança do público no sistema bancário, em decorrência de quebras de importantes instituições financeiras, como British Israel Bank (Inglaterra), Hersatt Bank (Alemanha), Franklin National Bank (EUA) e Banco Ambrosiano (Itália), que desencadeou a necessidade de se promoverem regulamentações, com a finalidade de melhorar a qualidade da informação disponibilizada ao usuário, de maneira que ele pudesse fazer a análise do risco do negócio. Essa regulamentação tomou corpo com a publicação do Acordo de Basiléia (Basiléia I, em 1988) e pelo Novo Acordo de Basiléia (Basiléia II, em 2004).

Um exemplo que ilustra a importância da transparência e da regulamentação para o adequado funcionamento do sistema financeiro é o fato de que, em reunião realizada no mês de março de 2009, ao realizar um diagnóstico da crise financeira vivenciada, o G-20² (2009), concluiu que: i) a fragilidade do disclosure teve um papel significante na crise; ii) as informações divulgadas não foram suficientes para muitos investidores e outros participantes do mercado; iii) as informações divulgadas nem sempre deixaram claro o tipo e a magnitude dos riscos, incluindo aqueles associados com exposições off-balance sheet; iv) as informações divulgadas sobre as exposições a risco de mercado e de crédito, particularmente em relação aos produtos estruturados foram deficientes; v) quando as informações eram divulgadas, não era feito de uma forma facilmente acessível; entre outras questões.

\subsection{Basiléia II e sua implementação no Brasil}

As orientações expostas em Basiléia II atualizam os padrões definidos no acordo de Basiléia I, buscando suprir suas deficiências e limitações, e apresentam alternativas mais sofisticadas para o cálculo do capital mínimo regulamentar, aproximando-o do capital econômico calculado pelos próprios bancos na gestão dos seus negócios.

Enquanto o acordo anterior exigia a alocação de capital para risco de crédito e risco de mercado, o novo referencial, além de promover mudanças conceituais relevantes em relação ao alcance do risco de mercado $^{3}$ e de considerar diferentes fatores de ponderação e mitigadores no cálculo da exposição ponderada pelo risco, também orienta a consideração dos riscos operacionais. Em relação às alternativas de cálculo de capital regulamentar, essas passam a considerar desde abordagens padronizadas, semelhantes ao padrão de Basiléia I, até

\footnotetext{
${ }^{2}$ Grupo dos 20 países mais ricos do mundo.

${ }^{3} \mathrm{O}$ risco de mercado segregou o risco de taxa de juros em quatro tipos diferentes (prefixado, cupom cambial, cupom de índice de preços e cupom de taxa de juros), mais a carteira banking. Também passou a considerar o risco de ações e commodities.
} 
abordagens avançadas e complexas, nas quais o capital regulatório é calculado a partir das próprias medidas de risco que os bancos utilizam internamente na gestão dos seus negócios.

Além do cálculo do capital regulamentar pela própria instituição, Basiléia II incorpora a revisão desse processo pela autoridade de supervisão bancária e a disciplina imposta pelo mercado, formando os três pilares que sustentam a nova estrutura regulatória:

- Pilar 1: Requerimentos de Capital: cálculo dos requerimentos de capital para risco de crédito, risco de mercado e risco operacional pelas próprias instituições financeiras, com base em metodologias mais sensíveis ao risco que as previstas por Basiléia I;

- Pilar 2: Revisão pela Supervisão: processo de autorização, revisão e validação pela supervisão bancária, assegurando a integridade das avaliações realizadas no Pilar 1;

- Pilar 3: Disciplina de Mercado: disciplina imposta pelo mercado, com a obrigatoriedade de divulgação de informações de maneira proporcional à complexidade das metodologias utilizadas para cálculo do próprio capital.

No Brasil, a implementação de Basiléia II ocorre em etapas, mediante a edição de normas definindo, inclusive, quais instituições podem optar pela abordagem avançada, com base em modelos internos de mensuração dos riscos de crédito, de mercado e operacional. Para a maioria das instituições, houve a sinalização de que deve ser aplicada a abordagem simplificada, que consiste em um aprimoramento da abordagem atual. Em relação ao Pilar 2 (processos de supervisão) e ao Pilar 3 (transparência e disciplina de mercado), o BCB ressalta que os requisitos serão aplicados a todas as instituições do SFN.

Sobre a relevância dessa estrutura normativa, Carvalho e Caldas (2009) afirmam que os países em desenvolvimento buscam implementar Basiléia II por acreditarem em um salto qualitativo na gestão, que poderia impactar, positivamente, nos bancos, por meio de menores riscos sistêmicos, menores spreads, elevação em seus ratings e outros.

Particularmente em relação ao Pilar 3, Doornik et al. (2007) destacam que é o estágio mais avançado para a redução dos riscos existentes no sistema financeiro, havendo, porém, um consenso sobre a dificuldade de se implantar a cultura da disciplina de mercado sobre os bancos, capaz de garantir os requisitos mínimos de transparência de informações.

\subsection{Pesquisas realizadas sobre evidenciação no SFN}

Nos últimos anos, alguns estudos têm procurado verificar o nível de evidenciação praticado pelas instituições do SFN, a maioria fazendo uma comparação com os requerimentos do Acordo de Basiléia II. 
Goulart (2003) analisou as demonstrações contábeis dos quatro maiores bancos brasileiros à época, referentes ao período de 1997 a 2002, e de um banco estrangeiro, dos anos de 2001 e 2002, com o objetivo de avaliar o grau de evidenciação contábil do risco de mercado no Brasil em comparação com levantamentos promovidos pelo Comitê de Basiléia sobre a evidenciação bancária no mercado internacional (BCBS, 2003). O estudo identificou indícios de evolução na evidenciação de informações de risco de mercado, porém concluiu que ela ainda é incipiente, quando comparada com as práticas de evidenciação internacionais. Além do mais, o baixo grau de detalhamento do conteúdo, segundo os autores, compromete a integralidade da divulgação.

Conclusão equivalente foi apresentada por Xavier (2003), ao avaliar o nível de transparência das demonstrações contábeis, dos anos de 2001 e 2002, dos dez maiores bancos brasileiros, em relação aos preceitos de Basiléia II e aos padrões de divulgação das instituições financeiras de atuação global.

Embora tendo por objetivo principal comparar os requerimentos de divulgação de Basiléia II à estrutura normativa do SFN, Ferreira e Araújo (2004) analisaram o nível de aderência das demonstrações de junho/2004 de três grandes instituições financeiras às recomendações do Acordo, constatando que, apesar de tais bancos se encontrarem em um patamar um pouco acima das exigências internas de evidenciação, ainda estão muito longe de atenderem às recomendações de Basiléia II.

Alves (2005) avaliou o nível de divulgação especificamente do risco operacional, no período de 2003 e 2004, dos 24 maiores bancos com carteira comercial que atuam no Brasil, concluindo que, embora a gestão desse risco estivesse em evolução, os resultados indicavam um longo caminho a ser percorrido para que a divulgação do risco operacional no contexto brasileiro seja considerada prática inclusiva no gerenciamento de riscos operacionais.

Tendo por objeto de estudo os relatórios anuais de 2001 a 2005 de três grandes instituições financeiras, o estudo de Di Benedito e Silva (2007) teve por foco verificar se houve evolução no nível de transparência no período analisado, em relação às recomendações do Novo Acordo de Basiléia. Inicialmente, os autores comparam os resultados de 2004 e 2005 com as conclusões da pesquisa de Xavier (2003), constatando uma melhoria nesse período, embora ainda baixa em relação à prática internacional. $\mathrm{Na}$ segunda parte do estudo, foi verificado se os bancos já estavam seguindo as recomendações constantes no Pilar III do Comitê de Basiléia, constatando-se um percentual médio de divulgação de apenas $20 \%$.

Outro trabalho equivalente foi realizado por Doornik et al. (2007), buscando verificar se as demonstrações contábeis publicadas pelos dez maiores bancos nacionais em volume de 
ativos (equivalente a 70\% do SFN), nos anos de 2003 a 2005, estavam em consonância com os critérios de divulgação estabelecidos em Basiléia II. Foram avaliados 104 itens de evidenciação e os resultados demonstraram que a maior parte dos itens não foi divulgada, tanto para bancos com ações negociadas em Bolsa, quanto para os de capital fechado. As conclusões da pesquisa mostraram que a divulgação das informações ainda se encontra num estágio incipiente, principalmente quando comparada aos bancos internacionais. Os motivos, segundo os autores, são a falta de um mercado financeiro que exerça concretamente poder disciplinador e a existência de lacunas na regulamentação brasileira em relação ao assunto.

Como síntese desses trabalhos realizados sobre o nível de evidenciação praticado pelas instituições do SFN, é possível afirmar que: i) embora em crescimento, o grau de evidenciação dos bancos brasileiros ainda é incipiente, quando comparado aos mercados mais desenvolvidos; ii) há uma dependência muito forte do arcabouço legal e normativo, o que induz a necessidade de revisão da estrutura regulamentar vigente e iii) as instituições brasileiras dão mais ênfase às informações quantitativas que às qualitativas.

\section{DESENVOLVIMENTO DAS HIPÓTESES DE PESQUISA}

Considerando os propósitos da pesquisa quanto à identificação de variáveis determinantes do grau de evidenciação do risco de crédito e, em particular, do impacto de Basiléia II nesse processo, são formuladas algumas hipóteses para os testes empíricos, tendo por referência relações sugeridas em estudos anteriores.

A primeira relação a ser estudada é o eventual impacto da divulgação de Basiléia II no nível de evidenciação do risco de crédito pelas instituições financeiras no Brasil, tendo em vista que, embora a sua implementação ainda esteja em processo, o BCB já comunicou que seguirá os requerimentos de transparência recomendados no Acordo. Essa premissa é baseada na afirmação de Admati e Pfleiderer (1998) de que a regulamentação do processo de evidenciação é fundamental para aumentar o grau de transparência do mercado e permitir o desenvolvimento da seguinte hipótese:

\section{$H_{1}$ : A vigência do Acordo de Basiléia II influenciou positivamente o comportamento das} instituições quanto à maior transparência das informações sobre o risco de crédito.

A percepção dos outsiders, uma das variáveis consideradas por Verrecchia (2001) para estudar a prática da divulgação voluntária, é utilizada como referência para se avaliar a relação entre o nível de evidenciação do risco de crédito e o tipo de controlador da instituição. O pressuposto é que instituições financeiras privadas, por não contarem com a garantia 
governamental, têm uma maior necessidade de demonstrar sua saúde financeira perante os depositantes, o que deve se refletir em um maior grau de divulgação. A hipótese resultante dessa perspectiva é assim formulada:

$\mathrm{H}_{2}$ : As instituições financeiras privadas apresentam maior nível de evidenciação sobre o risco de crédito que os bancos sob controle estatal.

Xavier (2003), Di Benedito e Silva (2007) e Doornik et al. (2007) compararam o padrão de evidenciação dos bancos brasileiros com as instituições financeiras internacionais, concluindo que o grau de transparência no mercado nacional é bem inferior ao verificado nos centros mais desenvolvidos. Partindo da premissa de que essa cultura de maior transparência por parte das instituições estrangeiras deve se revelar no mercado nacional, é formulada a seguinte hipótese a ser testada:

$H_{3}$ : As instituições financeiras sob controle de capital estrangeiro divulgam mais informações sobre risco de crédito que os bancos com controle nacional.

Um dos preceitos da teoria da divulgação voluntária de Verrechia (2001) é que o nível de divulgação da empresa tende a diminuir, na medida em que os custos relacionados com a evidenciação aumentam, refletindo a relação custo versus benefício de se divulgar. Estudos empíricos, como Salotti e Yamamoto (2008), utilizam o tamanho da empresa como proxy para a avaliação do impacto do custo de divulgação no nível de evidenciação. O pressuposto é que empresas maiores absorvem mais facilmente os custos de se produzirem informações, o que deve resultar em um maior grau de transparência. Nesse sentido, para os propósitos do presente estudo é formulada a seguinte hipótese:

$H_{4}$ : Os bancos de maior porte divulgam mais informações sobre risco de crédito que os de menor tamanho.

A relação positiva entre a performance da empresa e o nível de transparência praticado tem sido uma das principais questões avaliadas na literatura sobre disclosure e foi comprovada, por exemplo, por Watson et al. (2002) e por Eng e Mak (2003). Conforme a teoria da divulgação voluntária de Verrechia (2001), é esperado que empresas, com melhor desempenho, tenham mais incentivos para divulgar mais informações. Desse entendimento, resulta a seguinte hipótese a ser testada:

$H_{5}$ : Os bancos que apresentam melhores indicadores de rentabilidade e de gestão divulgam mais informações sobre risco de crédito.

Ao destacarem as formas que a evidenciação pode assumir, Ronen e Yaari (2002) destacam os mecanismos autoinduzidos de evidenciação, que se verificam quando as dinâmicas entre empresas e investidores forçam as companhias a divulgar as informações. É o Revista Contabilidade \& Finanças, USP, São Paulo, v. 21, n. 52, janeiro/abril 2010 
caso das empresas abertas, de quem são demandados padrões mais avançados de governança e de divulgação, inclusive por força regulamentar. Esse entendimento também é respaldado no preceito exposto por Verrechia (2001) de que o nível de divulgação tem correlação positiva com a importância dada pela empresa às percepções dos outsiders. Com isso, é formulada a seguinte hipótese de pesquisa:

$H_{6}:$ As instituições financeiras abertas, com ações listadas na Bovespa, divulgam mais informações sobre risco de crédito que as demais.

\section{METODOLOGIA}

A referência básica para os exames sobre o grau de evidenciação são as exigências previstas no Pilar 3 de Basiléia II para a divulgação de informações relativas a risco de crédito, em relação às instituições submetidas ao requerimento pelo método padronizado. Essa limitação se justifica pelo fato de que o $\mathrm{BCB}$ ainda não regulamentou o processo de acesso à opção pela abordagem avançada, com base em modelos internos de mensuração dos riscos. Sendo assim, serão considerados os requisitos gerais de divulgação qualitativa e quantitativa sobre o risco de crédito, bem como de sua mitigação, previstos em Basiléia II, conforme sintetizado nos Quadros 1 e 2, a seguir:

Quadro 1 - Risco de crédito - divulgações gerais para todos os bancos

\begin{tabular}{|c|c|l|}
\hline Tipo & Cod & \multicolumn{1}{|c|}{ Item de Divulgação } \\
\hline Divulgações & (a) & $\begin{array}{l}\text { Exigência de divulgação qualitativa geral (parágrafo 824) a respeito de risco de crédito, } \\
\text { incluindo: definições de itens vencidos e com valores reduzidos (para fins contábeis); } \\
\text { Descrição dos métodos usados para as provisões gerais e específicas e dos métodos } \\
\text { estatísticos; discussão da política de administração de riscos de crédito do banco. }\end{array}$ \\
\hline $\begin{array}{l}\text { Divulgações } \\
\text { Quantitativas }\end{array}$ & (b) & $\begin{array}{l}\text { Total bruto das exposições a risco de crédito mais a média da exposição bruta durante o } \\
\text { período, dividido pelos principais tipos de exposição de crédito. }\end{array}$ \\
\hline (c) & $\begin{array}{l}\text { Distribuição geográfica de exposições, desdobradas em áreas significativas pelos } \\
\text { principais tipos de exposição de crédito. }\end{array}$ \\
\hline (d) & $\begin{array}{l}\text { Distribuições de exposições do tipo do setor e das partes contratantes, desdobradas pelos } \\
\text { principais tipos de exposição de crédito. }\end{array}$ \\
\hline & (e) & $\begin{array}{l}\text { Desdobramento de vencimentos contratuais residuais de toda a carteira, desdobrados } \\
\text { pelos principais tipos de exposição de crédito. }\end{array}$ \\
\hline
\end{tabular}


(f)

provisões específicas e gerais; e encargos para provisões específicas e baixas durante o período.

Valor dos empréstimos deteriorados e, se disponível, empréstimos vencidos fornecidos

(g) separadamente, desdobrados pelas áreas geográficas significativas incluindo, se for praticável, os valores de provisões específicas e gerais relacionadas a cada área geográfica.

(h) Reconciliação de mudanças nas provisões para redução do valor de empréstimos.

Quadro 2 - Diminuição de risco de crédito - divulgações para os métodos padronizados

\begin{tabular}{|c|c|l|c|}
\hline Tipo & Cod & \multicolumn{1}{c|}{ Item de Divulgação } \\
\hline \multirow{2}{*}{$\begin{array}{c}\text { Divulgações } \\
\text { Qualitativas }\end{array}$} & (a) & $\begin{array}{l}\text { Exigência de divulgação qualitativa geral a respeito da diminuição de risco de crédito } \\
\text { incluindo: as políticas e processos para liquidação por compensação registrada e não } \\
\text { registrada no balanço patrimonial, e uma indicação da extensão na qual o banco faz o uso } \\
\text { dessa liquidação; as políticas e processos para administração e avaliação de caução; uma } \\
\text { descrição dos principais tipos de caução obtidas pelo banco; os principais tipos de } \\
\text { avalistas/partes contratantes de derivativos de crédito e sua capacidade financeira de } \\
\text { obter crédito; e as informações sobre concentrações de risco (de mercado ou crédito) } \\
\text { dentro da diminuição feita. }\end{array}$ \\
\hline Divulgações \\
Quantitativas
\end{tabular}

Fonte: Acordo de Basiléia II, artigos 824 a 826.

Considerando o exposto por Ferreira e Araújo (2004), de que os requisitos de divulgação no Brasil não contemplam os instrumentos de securitização de risco de crédito e que essa lacuna de regulamentação persiste nos dias atuais, esse tipo de requerimento não será contemplado no presente estudo.

\subsection{Seleção da Amostra}

Os testes realizados para o alcance dos objetivos estabelecidos têm como objeto de estudo as informações sobre o risco de crédito divulgadas por meio das IFT, no período entre 2001 e 2008, pelas organizações líderes dos conglomerados bancários ou instituições 
bancárias independentes que integram o relatório dos 50 Maiores Bancos do SFN, na posição de dezembro de 2008. Embora, pelo critério de seleção da amostra, os resultados obtidos não possam servir necessariamente como parâmetro para inferência sobre as demais instituições, a relevância da amostra pode ser justificada pelo fato de seus integrantes possuírem, em dezembro de 2008, o equivalente a 98,92\% dos ativos e a 99,04\% dos depósitos do SFN.

\subsection{Definição do Modelo de Análise}

Os trabalhos que avaliam o grau de evidenciação por parte das instituições financeiras, como BCBS (2003), Goulart (2003), Xavier (2003), Ferreira e Araújo (2004), Goulart e Carvalho (2004), Alves (2005), Di Benedito e Silva (2007), Doornik et al. (2007) e Alves e Sales (2008), trabalham, fundamentalmente, com uma análise das estatísticas descritivas, analisando a evolução da média de divulgação ao longo de determinado período ou fazendo uma comparação com um determinado benchmark ou situação desejável.

Este estudo avalia o nível de evidenciação com a utilização de regressão linear, que, segundo Brooks (2008), é a mais importante ferramenta para descrever e avaliar a relação entre variáveis. No caso específico, o propósito é identificar que variáveis podem explicar o nível de evidenciação de risco de crédito pelas instituições financeiras no Brasil.

Nesse sentido, a análise dos dados terá como referência o modelo a seguir descrito, elaborado a partir dos propósitos do estudo e das hipóteses formuladas no capítulo 3 :

$$
D R C_{i, t}=\alpha+\beta_{1} B A S_{i, t}+\beta_{2} T C_{i, t}+\beta_{3} N C+\beta_{4} \ln T A M_{i, t}+\beta_{5} R E N T_{i, t}+\beta_{6} I B_{i, t}+\beta_{7} I I_{i, t}+\beta_{8} B O V_{i, t}+\varepsilon_{i, t}
$$

em que:

- $D R C_{i, t}$ é o nível de evidenciação do risco de crédito da instituição $i$, no período $t$, apurado na pesquisa a partir da análise de conteúdo das IFT de cada instituição entre 2001 e 2008, tendo como referência os requisitos de divulgação previstos em Basiléia II, conforme Quadros 1 e 2;

- $\quad B A S_{i, t}$ é uma variável do tipo dummy para a identificação da vigência de Basiléia II, sendo definida como 0 para as IFT referentes aos anos de 2001 a 2004 e como 1 para as de 2005 a 2008;

- $\quad T C_{i, t}$ é uma variável do tipo dummy para a identificação do tipo do controlador, sendo definida como 1 para o controle privado e 0 para o controlador estatal (da amostra, 41 instituições são privadas e 9 estatais);

- $\quad N C_{i, t}$ é uma variável do tipo dummy para a identificação da nacionalidade de origem 
do capital de controle, sendo definida como 1 para o capital nacional e 0 para o capital estrangeiro (da amostra, 30 instituições têm controle de capital nacional e 20 de capital estrangeiro);

- $\quad T A M_{i, t}$ é uma medida que representa o tamanho da instituição $i$ no período $t$, apurada pela relação entre os ativos totais menos intermediação financeira e a soma desses ativos em todo o segmento, apurado e divulgado pelo BCB;

- $\quad R E N T_{i, t}$ traduz a rentabilidade da instituição $i$, no período $t$, definida como a relação entre o lucro líquido e o patrimônio líquido ajustado (deduzido do lucro ou prejuízo líquido do período) em cada período examinado;

- $\quad I B_{i, t}$ traduz o índice de Basiléia da instituição $i$, no período $t$, apurado e divulgado pelo $\mathrm{BCB}$;

- $\quad I I_{i, t}$ é o índice de imobilização da instituição $i$, no período $t$, apurado e divulgado pelo $\mathrm{BCB}$;

- $\quad B O V_{i, t}$ é uma variável dummy que indica se a instituição $i$, no período $t$, tinha ações listadas na Bovespa, sendo definida como 0 as empresas não listadas e 1 as listadas (da amostra, 22 instituições têm ações listadas na Bovespa e 28 não);

- $\quad \beta_{1,2,3, . .8}$ representam os coeficientes de cada uma das respectivas variáveis independentes;

- $\quad \alpha$ é o coeficiente do intercepto da regressão e

- $\quad \varepsilon_{i, t}$ é o termo de dispersão ou erro da regressão, relacionado à instituição $i$, no período $t$, assumindo a normalidade dos resíduos, com média zero, variância constante e ausência de correlação, ou seja, $\sim \mathrm{N}\left(E\left(\varepsilon_{i, t}\right)=0, \operatorname{Var}\left(\varepsilon_{i, t}\right)=\sigma^{2}\right.$ e $\left.\operatorname{Cor}\left(\varepsilon_{i,} \varepsilon_{j}\right)=0\right)$.

A relação entre as variáveis independentes do modelo e as hipóteses de pesquisa a serem testadas, é demonstrada no Quadro 3.

Quadro 3 - Associação entre as hipóteses de pesquisas e as variáveis independentes do modelo

\begin{tabular}{|c|c|c|c|c|c|c|}
\hline Hipóteses & $H_{1}$ & $H_{2}$ & $H_{3}$ & $H_{4}$ & $H_{5}$ & $H_{6}$ \\
\hline Variáveis & $B A S$ & $T C$ & $N C$ & $T A M$ & $R E N T, I B, I I$ & $B O V$ \\
\hline
\end{tabular}

No caso específico da apuração da variável $D R C_{i, t}$, o procedimento para apuração da variável consistiu em atribuir notas entre 0 (quando não atende ao requisito de divulgação) e 1 (quando atende completamente à exigência de evidenciação) para cada um dos itens 
constantes dos Quadros destacados. Para os casos em que há atendimento parcial ao estabelecido por Basiléia II para aquele item, são atribuídas notas intermediárias proporcionais aos subitens observados.

A partir dessa avaliação em relação a cada item de divulgação, descritos nos Quadros 1 e 2, é apurado o grau de divulgação do risco de crédito da instituição $i$, no período $t$, de acordo com a seguinte fórmula:

$$
D R C_{i, t}=\frac{\sum_{Q 1=a}^{h} N_{Q 1_{i, t}}+\sum_{Q 2=a}^{c} N_{Q 2_{i, t}}}{11}
$$

em que: $N_{Q 1}$ representa a nota apurada em relação a cada um dos itens de divulgação "a" a "h" do Quadro 1 e $N_{Q 2}$ a nota apurada nos itens de divulgação "a" a "c" do Quadro 2.

Tendo em vista que há uma subjetividade implícita nesse processo de apuração da variável $D R C$, foi adotado o procedimento de análise de cada demonstração por pelo menos dois pesquisadores, com o fim de reduzir os efeitos da subjetividade do exame e o eventual viés inerente a esse tipo de estudo: análise de conteúdo. Isso, obviamente, não elimina a subjetividade do processo, o que se constitui em limitação do estudo.

\subsection{Procedimentos de testes das hipóteses}

A partir dos preceitos das hipóteses de pesquisa, espera-se que os parâmetros das variáveis explicativas do modelo sejam estatisticamente relevantes para explicar o grau de evidenciação do risco de crédito. Quanto ao sinal, é esperado que as variáveis $B A S, T C$, $T A M, R E N T, I B$ e $B O V$ apresentem sinal positivo, enquanto os parâmetros das variáveis $N C$ e $I I$ sinal negativo.

Para a realização dos testes, o modelo desenvolvido é aplicado com a metodologia de dados em painel, que considera o comportamento das variáveis tanto na dimensão temporal quanto na espacial. Baltagi (2008) e Marques (2000) apresentam uma série de vantagens da estimação com dados em painel, entre as quais podem ser destacados: o controle da heterogeneidade individual; o provimento de dados com maior poder de informação, maior variabilidade, menor colinearidade entre as variáveis, maior número de graus de liberdade e maior eficiência estatística na estimação; a possibilidade de se facilitar uma análise mais eficiente das dinâmicas de ajustamento; a possibilidade de se identificar e medir efeitos que não são detectáveis em estudos exclusivamente seccionais ou temporais; entre outras.

Associando-se esses benefícios às características da pesquisa, em que são 
considerados aspectos seccionais (amostra composta dos 50 maiores bancos) e temporais (comportamento de oito variáveis independentes e do grau de evidenciação do risco de crédito ao longo do período), fica evidenciada a relevância da utilização dos dados em painel para aumentar a eficiência estatística dos testes realizados neste estudo.

Considerando a dinâmica do presente estudo, em que o objetivo é identificar as variáveis que explicam o nível de transparência quanto ao risco de crédito dos bancos brasileiros, são utilizados os dois modelos de dados em painel: (i) modelo de efeitos fixos: pressupõe que as inclinações se mantêm, mas os interceptos podem variar entre os elementos da amostra ou no tempo, ainda que permaneçam como constantes fixas e (ii) modelo de efeitos aleatórios: trata os efeitos individuais do intercepto, destacados no modelo de efeitos fixos, como se termos de perturbação fossem, isto é, especifica os efeitos individuais não de forma determinística, mas aleatória. Com isso, pretende-se verificar se as hipóteses da pesquisa podem ser corroboradas, independentemente do modelo utilizado. A opção por realizar os testes com os modelos de efeitos fixos e de efeitos aleatórios deve-se ao propósito de verificar a relevância das variáveis independentes para explicar o comportamento do grau de evidenciação do risco de crédito, independentemente do método de dados em painel adotado. Configura-se, portanto, um parâmetro de robustez das evidências obtidas.

\section{APURAÇÃO E ANÁLISE DOS DADOS}

Utilizando como referência o modelo descrito na seção 4.2, foram apuradas as variáveis dos 50 bancos integrantes da amostra, considerando o período entre o primeiro trimestre de 2001 e o quarto de 2008 , tendo por base as seguintes fontes: i) a variável $D R C$ foi apurada com base nas IFT disponibilizadas no sítio do $\mathrm{BCB}$; ii) as variáveis $T C, N C$, $T A M, R E N T, I B$ e $I I$ foram apuradas ou obtidas diretamente no Relatório dos 50 maiores bancos, também disponibilizado pelo Banco Central; iii) a variável $B O V$ foi constituída com base nas informações do relatório Classificação Setorial das Empresas e Fundos Negociados na BM\&FBovespa, disponível no sítio da instituição na internet e iv) a variável $B A S$ é considerada a partir da data de referência de divulgação do Acordo de Basiléia II.

\subsection{Apuração do nível de divulgação do risco de crédito}

Inicialmente, foi promovida a apuração da variável $D R C$, que mede o grau de evidenciação do risco de crédito e constitui o foco do estudo, apurado em função dos 
requisitos de divulgações qualitativas e quantitativas estabelecidas por Basiléia II, descritas nos Quadros 1 e 2, conforme metodologia exposta na seção 4.2. O resultado médio apurado, considerando o conjunto das instituições, em cada trimestre é consolidado na Tabela 1.

Tabela 1 - Média da variável $D R C$ apurada em cada semestre, considerando o conjunto das instituições

\begin{tabular}{|c|c|c|c|c|c|c|c|c|c|c|c|}
\hline Ano & Trim & $\overline{D R C}$ & Ano & Trim & $\overline{D R C}$ & Ano & Trim & $\overline{D R C}$ & Ano & Trim & $\overline{D R C}$ \\
\hline \multirow{4}{*}{2001} & $1^{\circ}$ & 0.3365 & \multirow{4}{*}{2003} & $1^{\circ}$ & 0.3518 & \multirow{4}{*}{2005} & $1^{\circ}$ & 0.3578 & \multirow{4}{*}{2007} & $1^{\circ}$ & 0.3698 \\
\hline & $2^{\circ}$ & 0.3387 & & $2^{\circ}$ & 0.3515 & & $2^{\circ}$ & 0.3578 & & $2^{\circ}$ & 0.3733 \\
\hline & $3^{\circ}$ & 0.3387 & & $3^{\circ}$ & 0.3510 & & $3^{\circ}$ & 0.3567 & & $3^{\circ}$ & 0.3738 \\
\hline & $4^{o}$ & 0.3422 & & $4^{\circ}$ & 0.3515 & & $4^{\circ}$ & 0.3567 & & $4^{o}$ & 0.3808 \\
\hline \multirow{4}{*}{2002} & $1^{\circ}$ & 0.3483 & \multirow{4}{*}{2004} & $1^{\circ}$ & 0.3522 & \multirow{4}{*}{2006} & $1^{\circ}$ & 0.3613 & \multirow{4}{*}{2008} & $1^{\circ}$ & 0.3838 \\
\hline & $2^{\circ}$ & 0.3448 & & $2^{\circ}$ & 0.3522 & & $2^{\circ}$ & 0.3633 & & $2^{\circ}$ & 0.3851 \\
\hline & $3^{\circ}$ & 0.3438 & & $3^{\circ}$ & 0.3533 & & $3^{\circ}$ & 0.3638 & & $3^{\circ}$ & 0.3856 \\
\hline & $4^{o}$ & 0.3460 & & $4^{o}$ & 0.3535 & & $4^{o}$ & 0.3655 & & $4^{o}$ & 0.3904 \\
\hline
\end{tabular}

A análise dos valores médios da variável $D R C$, ao longo dos 32 trimestres analisados, permite concluir que o nível de evidenciação do risco de crédito pelos bancos brasileiros aumentou de forma constante e paulatina, embora ainda esteja em patamar muito baixo, quando comparado aos requisitos de Basiléia II. Conforme o Gráfico I, o nível de evidenciação partiu de $33,65 \%$ de atendimento dos requisitos de divulgação previstos em Basiléia II no primeiro trimestre de 2001 até atingir 39,04\% no quarto trimestre de 2008.

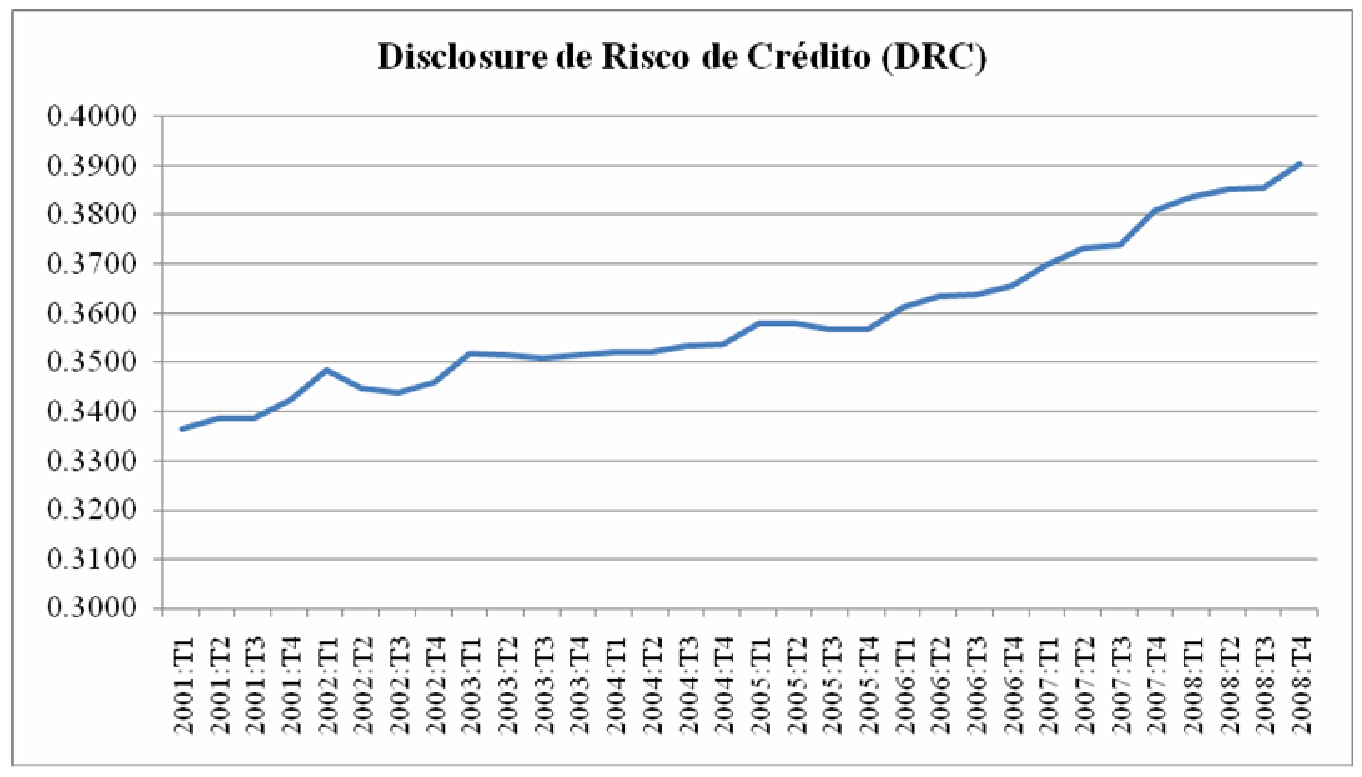

Gráfico I - Evolução do grau de evidenciação de risco de crédito, no período de 2001 a 2008

O comportamento de contínuo crescimento demonstra que o grau de evidenciação, em um período $t$, está diretamente relacionado à divulgação praticada no período imediatamente 
anterior, ou seja, a prática das instituições ao divulgar suas informações sobre o risco de crédito tem como referência principal a divulgação no período anterior, promovendo algumas informações adicionais, quando for o caso.

\subsection{Análise dos Determinantes do Grau de Evidenciação}

Para apurar a relevância das variáveis independentes na explicação do grau de evidenciação do risco de crédito, foram realizados testes com os modelos de efeitos fixos e de efeitos aleatórios cross-section, cujos resultados são consolidados na Tabela 2:

Tabela 2 - Resultados da regressão: dados em painel, com efeitos fixos e com efeitos aleatórios

\begin{tabular}{|c|c|c|c|c|c|c|c|}
\hline \multicolumn{2}{|c|}{ Variável dependente: } & \multicolumn{2}{|l|}{$D R C$} & & & & \\
\hline & \multicolumn{2}{|c|}{ 2001:1 a 2008:4 } & & & & \\
\hline \multicolumn{2}{|c|}{$\mathrm{N}^{\mathrm{o}}$ de empresas: } & 50 & & & & & \\
\hline \multicolumn{2}{|c|}{$\mathrm{N}^{\mathrm{o}}$ de trimestres: } & 32 & & & & & \\
\hline \multicolumn{2}{|c|}{$\mathrm{N}^{\mathrm{o}}$ de observações: } & 1.599 & & & & & \\
\hline \multicolumn{4}{|c|}{ Método: Efeitos fixos (cross) ${ }^{4}$} & \multicolumn{4}{|c|}{ Método: Efeitos aleatórios (cross) } \\
\hline Variável & Coef. & t-Stat & p-valor & Variável & Coef. & t-Stat & p-valor \\
\hline$C$ & 0.42326 & 31.70388 & 0.0000 & $C$ & 0.42611 & 21.94326 & 0.0000 \\
\hline$B A S$ & 0.01885 & 10.17281 & 0.0000 & $B A S$ & 0.01940 & 10.54600 & 0.0000 \\
\hline $\ln (T A M)$ & 0.01284 & 5.52622 & 0.0000 & $\ln (T A M)$ & 0.01181 & 6.15216 & 0.0000 \\
\hline RENT & 0.03391 & 4.10410 & 0.0000 & $R E N T$ & 0.03463 & 4.20670 & 0.0000 \\
\hline II & -0.02623 & -3.00257 & 0.0027 & II & -0.01971 & -2.30237 & 0.0214 \\
\hline \multirow[t]{4}{*}{$I B$} & 0.00005 & 0.99544 & 0.3197 & $I B$ & 0.00002 & 0.53242 & 0.5945 \\
\hline & & & & $T C$ & -0.02149 & -1.48301 & 0.1383 \\
\hline & & & & $N C$ & -0.00680 & -0.55021 & 0.5823 \\
\hline & & & & $B O V$ & 0.02614 & 2.10061 & 0.0358 \\
\hline$R^{2}:$ & 0.7564 & F-stat: & 88.798 & $R^{2}:$ & 0.1395 & F-stat: & 32.2232 \\
\hline$\overline{R^{2}}:$ & 0.7479 & $\operatorname{Prob}(\mathrm{F})$ & 0.0000 & $\overline{R^{2}}:$ & 0.1352 & $\operatorname{Prob}(\mathrm{F}):$ & 0.0000 \\
\hline
\end{tabular}

Os resultados dos testes com efeitos fixos e com efeitos aleatórios mostram que, das variáveis explicativas consideradas no modelo utilizado como referência, apresentaram significância estatística os parâmetros das variáveis referentes à vigência de Basiléia II $(B A S)$, ao tamanho das instituições (TAM), à rentabilidade dos bancos (RENT), ao índice

\footnotetext{
${ }^{4}$ Considerando a natureza das variáveis $T C, N C$ e $B O V$ - tipo dummy, com variações cross-section - não é tecnicamente possível considerá-las em um modelo com dados em painel com efeitos fixos cross-section. Por essa razão, só são consideradas no modelo com efeitos aleatórios. 
de imobilização (II ) e à condição de ter capital aberto ( $B O V)$, conforme as estatísticas $t$ e os p-valor destacados na Tabela 2.

Para identificar quais variáveis são determinantes no grau de evidenciação do risco de crédito por parte dos bancos brasileiros, é promovido o cotejamento desses resultados da regressão com as hipóteses de pesquisa destacadas no capítulo 3.

No caso da hipótese $H_{1}$, a relevância da variável $B A S$ para explicar o comportamento das instituições em divulgar informações sobre o risco de crédito, conforme evidenciado pelas estatísticas $t$ e pelos p-valor correspondentes, tanto no método de efeitos fixos quanto no método de efeitos variáveis, é uma evidência de que a vigência do Acordo de Basiléia II influenciou positivamente no padrão de transparência dos bancos brasileiros. Em resumo, o padrão de evidenciação do risco de crédito a partir de 2005 é significativamente maior que no período entre 2001 e 2004. Considerando que após a edição do novo Acordo, o BCB não alterou a regulamentação que disciplina a evidenciação do risco de crédito, é possível inferirse que essa melhoria contínua, embora ainda distante de alcançar o patamar requerido, tenha sido impactada pela divulgação da importância que Basiléia II atribui aos requisitos de transparência e disciplina de mercado. Em suma, os resultados corroboram $H_{l}$.

Em relação à hipótese $H_{2}$, os resultados não apresentam evidências de que as instituições privadas apresentem um melhor nível de evidenciação de risco de crédito de que os bancos públicos. A variável $T C$ não apresentou significância estatística, ou seja, não há diferença no grau de transparência entre os bancos sob controle privado ou público, rejeitando-se a hipótese de pesquisa.

Situação semelhante é verificada em relação à variável $N C$, que avalia a diferença de comportamento na divulgação sobre o risco de crédito por instituições cujo controle de capital seja nacional ou estrangeiro. A variável não se mostrou relevante para explicar a evidenciação, ou seja, não se identificou diferença de comportamento quanto à transparência das instituições, com base na nacionalidade do capital de controle. Assim, de forma equivalente à hipótese $\mathrm{H}_{2}, \mathrm{H}_{3}$, também, é rejeitada. Essas evidências indicam que, embora nos seus países de origem as instituições financeiras internacionais pratiquem um nível de divulgação mais elevado que o registrado no mercado brasileiro, conforme destacado por Xavier (2003), Di Benedito e Silva (2007) e Doornik et al. (2007), no Brasil apresentam um comportamento equivalente às instituições nacionais, ou seja, não praticam internamente a cultura de transparência que exercem nos seus países de origem. 
A hipótese $H_{4}$ considera a premissa de que os bancos de maior porte divulgam mais informações sobre o risco de crédito. Os resultados das estatísticas $t$ e os $p$-valor demonstram que o comportamento da variável TAM corrobora a hipótese, ou seja, a divulgação de informações sobre o risco de crédito pelos bancos que possuem maior volume de ativos é maior que a praticada pelos bancos menores, corroborando-se a hipótese de estudo.

No caso da hipótese $H_{5}$, o esperado é que as instituições com os melhores indicadores de rentabilidade e de gestão divulguem mais informações sobre o risco de crédito. Nesse particular, os resultados obtidos em relação às variáveis RENT e II corroboram a hipótese de pesquisa. Há relação positiva estatisticamente significativa entre o grau de transparência e o nível de rentabilidade e uma relação negativa, também relevante, entre o índice de imobilização e o nível de evidenciação de risco de crédito. Não foi confirmada, porém, a esperada relação positiva entre o grau de evidenciação e o índice de Basiléia da instituição. A variável $I B$ não se mostrou relevante para explicar o padrão de transparência adotado pelos bancos brasileiros. Dessa forma, a hipótese $H_{5}$ é corroborada em relação ao nível de rentabilidade e ao índice de imobilização, mas é rejeitada em relação ao índice de Basiléia, apurado pelo BCB.

Por fim, a hipótese $H_{6}$ assume a premissa de que entidades com ações listadas em Bolsa de Valores adotam um maior nível de divulgação, considerando os requisitos de governança e de transparência a que são submetidos pelas regras do mercado de capitais, bem como à demanda dos investidores. As evidências obtidas no estudo, com a relevância estatística da variável $B O V$ na explicação da variável dependente $D R C$, confirmam essa hipótese da pesquisa.

\subsection{Teste adicional - análise do impacto dos valores extremos}

Como um procedimento de verificação adicional de robustez dos achados, foi realizado novo teste, tendo por referência o mesmo modelo, mas retirando-se da base de dados os outliers (considerados como aqueles que se desviam mais ou menos três desvios padrões em relação à média) de todas as variáveis. O objetivo é identificar se os resultados destacados na seção 5.2 não teriam sido determinados pelo comportamento de valores extremos, o que poderia configurar uma limitação das evidências obtidas. Os resultados desse teste adicional são consolidados na Tabela 3 (a exclusão dos outliers reduziu o número de observações para 1.484): 
Tabela 3 - Resultados da regressão sem outliers: dados em painel, com efeitos fixos e com efeitos aleatórios

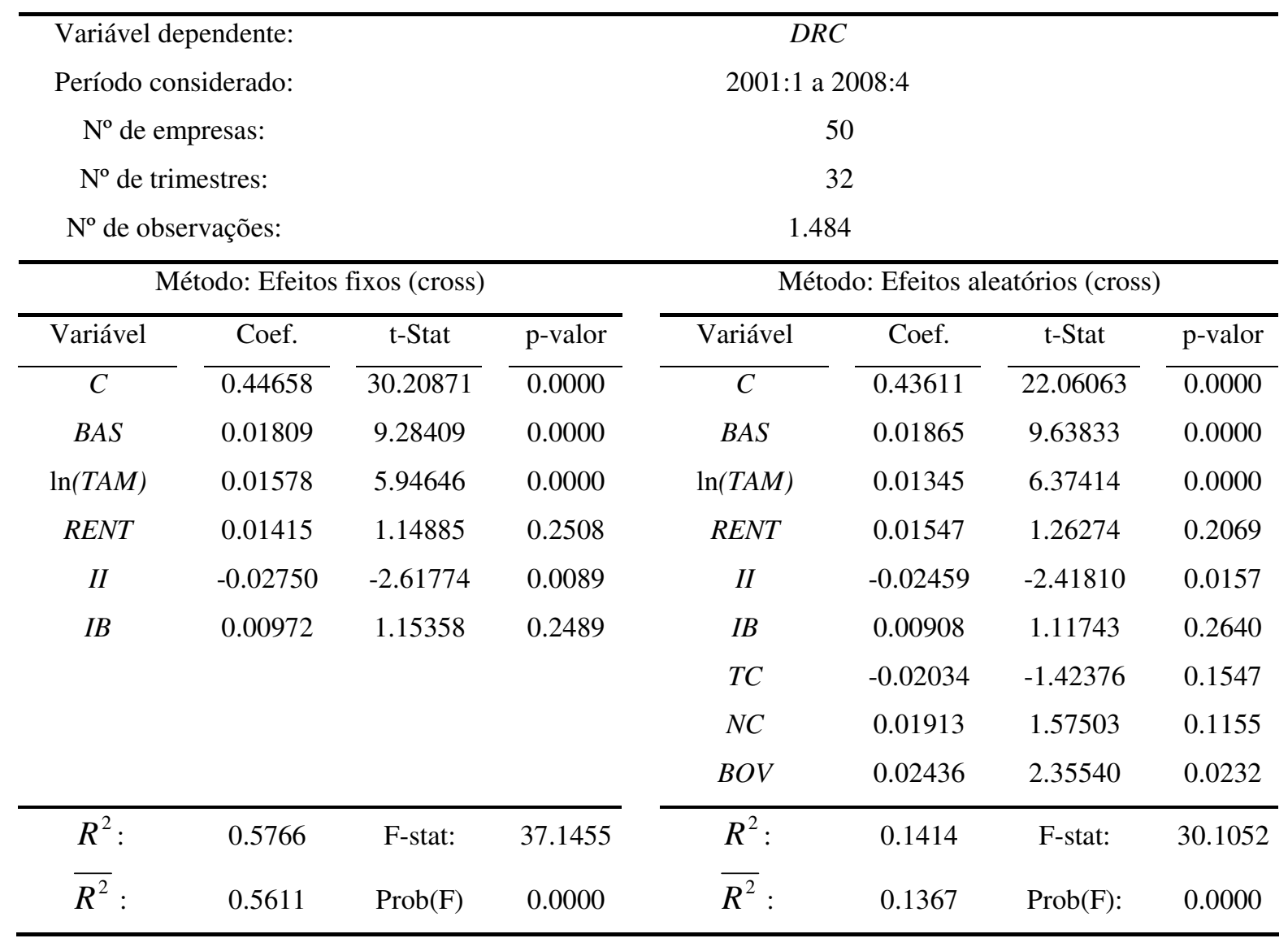

Como se depreende dos dados da Tabela 3, em comparação com os da Tabela 2, os resultados obtidos com a base de dados sem os outliers são praticamente equivalentes aos obtidos com a base completa, sintetizados da seguinte forma:

- as variáveis $B A S, T A M, I I$ e $B O V$ apresentam comportamento semelhante ao verificado quando se considerou a amostra completa, seja quanto à significância estatística ou ao sinal, com pequenas variações na dimensão das estatísticas $t$ e nos $p$-valor, tanto no modelo de efeitos fixos quanto no de efeitos variáveis;

- a variável RENT, relevante ao se considerar a base completa, tanto no modelo de efeitos fixos quanto no de efeitos variáveis, não se mostrou significante ao serem excluídos os valores extremos, também nos dois modelos, ou seja, o nível de rentabilidade dos bancos só é relevante para explicar o grau de transparência em relação ao risco de crédito, se forem consideradas as situações extremas e

- $\quad$ as variáveis $T C, N C$ e $I B$, que não apresentavam significância estatística ao se considerar a base completa, continuaram sem ser relevante ao se excluir os outliers.

Esses resultados reforçam as conclusões do estudo sobre a significância das variáveis $B A S, T A M, I I$ e $B O V$ para explicar o comportamento do grau de evidenciação do risco de 
crédito por parte dos bancos brasileiros. No caso da variável representativa do nível de rentabilidade das instituições, porém, ficou evidenciado que sua relevância para explicar a transparência do risco de crédito depende, essencialmente, da consideração de situações extremas, o que reduz a sua importância para a conclusão do presente estudo.

\subsection{Análise da Significância do Modelo}

Os coeficientes de determinação da regressão, $R^{2}$ e $\overline{R^{2}}$, que são medidas resumidas do quanto a linha de regressão amostral se ajusta aos dados, alcançam 0,7564 e 0,7479, respectivamente, no caso do método com efeitos fixos, e 0,1395 e 0,1352 no método com efeitos variáveis, quando se considera o total das observações da amostra. Ao se excluirem os outliers, o $R^{2}$ e o $\overline{R^{2}}$ assumem 0,5766 e 0,5611 , no modelo com efeitos fixos, e 0,1574 e 0,1467, no método com efeitos variáveis, respectivamente.

Essa divergência do nível de coeficiente de determinação entre as regressões com efeitos fixos e efeitos aleatórios se justifica pela diferença metodológica entre os dois mecanismos. Enquanto nos efeitos fixos a heterogeneidade dos efeitos individuais é considerada como constantes fixas adicionais, no modelo com efeitos variáveis essa heterogeneidade individual é incorporada no termo de distúrbio ou erro. Por essa razão, o coeficiente de determinação com o método de efeitos variáveis é bem menor que no método com efeitos fixos.

Outra medida da relevância do modelo é demonstrada pelo $F$-test, que demonstra a significância estatística do conjunto dos parâmetros das variáveis independentes na explicação do comportamento da variável dependente. Conforme destacado nas Tabelas 2 e 3, a F-stat varia entre 30,1052 e 88,7989, rejeitando-se a hipótese nula de insignificância dos parâmetros, com p-valor 0,0000 , em todos os cenários examinados: com a amostra completa ou excluindo os outliers, utilizando o método com efeitos fixos ou com efeitos aleatórios.

Por fim, foi realizado o teste sugerido por Baltagi (2008) e Marques (2000), para demonstrar a existência de efeitos individuais, o que justifica a utilização dos dados em painel. É uma versão do teste de Chow, tendo em vista a similaridade com o conhecido teste de estabilidade dos parâmetros:

$$
C H O W=\frac{(R R S S-U R S S) /(N-1)}{U R S S /(N T-N-K)} \sim F_{(N-1, N T-N-K)}
$$

em que: RRSS é a soma dos quadrados dos resíduos considerando o modelo pooled (desconsiderando a heterogeneidade individual dos elementos); URSS a soma dos quadrados 
dos resíduos com o modelo de efeitos fixos; $N$ o número de empresas integrantes da amostra; $T$ o número de períodos considerados no estudo e $K$ o número de variáveis, incluindo a constante.

A estatística apurada é comparada com a distribuição $F$, conforme Tabela 4, resultando na rejeição da hipótese nula de que os resultados apurados sem a consideração da heterogeneidade individual seriam apropriados. Demonstra, portanto, a relevância da utilização dos dados em painel para o provimento de dados com maior poder informacional.

Tabela 4 - Resultados dos testes para heterogeneidade não observada

\begin{tabular}{cccc}
\hline CHOW Stat & $F_{(49,1.544,5 \%)}$ & $F_{(49,1.544,1 \%)}$ & Conclusão \\
\hline 265,86 & 1,50 & 1,70 & Rejeita $\mathrm{H}_{0}-$ há heterogeneidade individual. \\
\hline
\end{tabular}

\subsection{Teste de estacionariedade das séries}

Para testar a estacionariedade das séries, ou seja, identificar a presença de raízes unitárias, foram utilizados os testes Im, Pesaran e Shin (I.P.S.), ADF-Fisher e PP-Fisher. Os testes de raízes unitárias foram realizados em relação às séries $D R C, \ln (T A M), R E N T, I B$ e II . Para as variáveis $B A S, T C, N C$ e $B O V$, por serem variáveis binárias, não se aplicam esses testes. Os resultados estão consolidados na Tabela 5:

Tabela 5 - Resultados dos testes para raízes unitárias

\begin{tabular}{|c|c|c|c|c|c|c|c|}
\hline & \multirow[t]{2}{*}{ Variável } & \multicolumn{2}{|c|}{ Teste I.P.S. } & \multicolumn{2}{|c|}{ ADF Fisher } & \multicolumn{2}{|c|}{$P P \quad$ Fisher } \\
\hline & & Stat & $p$-valor & Stat & $p$-valor & Stat & $p$-valor \\
\hline \multirow{5}{*}{$\begin{array}{l}\text { Amostra } \\
\text { completa }\end{array}$} & $D R C$ & -10.8705 & $\overline{0.0000}$ & 63.4179 & 0.2913 & 127.9070 & $\overline{0.0009}$ \\
\hline & $\ln (T A M)$ & -0.9948 & 0.1599 & 119.9210 & 0.0852 & 158.2440 & 0.0002 \\
\hline & RENT & -13.1549 & 0.0000 & 397.4970 & 0.0000 & 844.0200 & 0.0000 \\
\hline & $I B$ & -9.3323 & 0.0000 & 289.3830 & 0.0000 & 318.8200 & 0.0000 \\
\hline & $I I$ & -2.3663 & 0.0090 & 149.9600 & 0.0009 & 198.4460 & 0.0000 \\
\hline \multirow{5}{*}{$\begin{array}{c}\text { Sem } \\
\text { outliers }\end{array}$} & $D R C$ & -10.8705 & 0.0000 & 63.4179 & 0.2913 & 127.9070 & 0.0009 \\
\hline & $\ln (T A M)$ & -1.5409 & 0.0617 & 127.2820 & 0.0250 & 160.1660 & 0.0001 \\
\hline & RENT & -12.8899 & 0.0000 & 392.3620 & 0.0000 & 887.5080 & 0.0000 \\
\hline & $I B$ & -13.8752 & 0.0000 & 281.0480 & 0.0000 & 330.8000 & 0.0000 \\
\hline & $I I$ & -1.3133 & 0.0945 & 124.5700 & 0.0486 & 186.2150 & 0.0000 \\
\hline
\end{tabular}

A combinação dos resultados dos três tipos de testes revela que a hipótese nula da existência de raízes unitárias para cada série é rejeitada em pelo menos dois dos três testes. 
Isso demonstra não haver evidências de raízes unitárias, eliminando-se o risco de regressões espúrias.

\section{CONCLUSÃO}

O presente estudo teve o objetivo de avaliar o grau de transparência praticado pelas instituições financeiras brasileiras, tendo como referência as divulgações qualitativas e quantitativas requeridas por Basiléia II para o risco de crédito. O pressuposto foi de que, apesar de estar em fase de implementação no Brasil, os pronunciamentos e as diretrizes publicados poderiam influenciar o comportamento dos bancos brasileiros em suas divulgações, considerando ainda o fato de que o BCB comunicou a intenção de adotar os requisitos do Pilar 3 para todas as instituições.

Para a realização dos testes empíricos, foram selecionados os 50 maiores bancos na posição de dezembro de 2008 e analisadas as Informações Financeiras Trimestrais entre 2001 e 2008. Os dados empíricos demonstraram que o grau de evidenciação do risco de crédito tem aumentado de forma constante e paulatina entre o primeiro trimestre de 2001 e o quarto de 2008, mas ainda está distante do padrão requerido por Basiléia II. Embora em crescimento, o índice médio de divulgação dos requerimentos de divulgação de Basiléia II para o risco de crédito foi de apenas 39,04\% ao final de 2008. No primeiro trimestre de 2001 era de 33,65\%. Isso pressupõe a necessidade de um esforço tanto das próprias instituições financeiras como regulamentar, para que o grau de transparência previsto no Pilar 3 seja alcançado.

Também foi constatado que o nível de evidenciação de risco de crédito de um período é influenciado pelo que foi reportado no período imediatamente anterior. Ao divulgar uma determinada informação, a prática comum é voltar a divulgá-la, agregando, quando for o caso, alguma informação nova, que passa a ser a nova referência para a divulgação do momento seguinte.

No que concerne à identificação das variáveis determinantes do nível de divulgação sobre o risco de crédito praticado pelos bancos brasileiros, foram realizados testes, a partir de um modelo de regressão construído para verificar as seis hipóteses que associavam o grau de transparência às características específicas julgadas importantes para explicar as eventuais diferenças de divulgação.

Os testes, realizados com o uso da metodologia de dados em painel, corroboraram as hipóteses de que a vigência do Acordo de Basiléia II modificou o padrão de evidenciação e de que os bancos com maior volume de ativos, com maior nível de rentabilidade, menor 
imobilização e com ações listadas na Bovespa apresentam maior grau de transparência sobre o risco de crédito.

Por outro lado, não foram confirmadas as hipóteses de que os bancos privados, com controle de capital estrangeiro e com índice de Basiléia mais elevado apresentariam grau de evidenciação maior do que os bancos públicos, com controle de capital nacional e com menor índice de Basiléia, respectivamente. Os testes demonstraram que os padrões de evidenciação praticados por essas instituições não são significativamente diferentes entre si para explicar o comportamento da variável dependente.

Com o fim de atestar a consistência dos achados, foram realizados testes adicionais, dessa vez com a exclusão dos outliers das diversas séries das bases de dados, obtendo-se resultados equivalentes aos inicialmente encontrados, exceto pelo fato de que a variável representativa da rentabilidade das instituições deixou de ser significativamente relevante para explicar o grau de divulgação do risco de crédito por parte dos bancos brasileiros.

Para confirmar a relevância dos testes empíricos, em particular da aplicação da metodologia de dados em painel, foi realizado o teste de Chow, confirmando-se sua relevância para o provimento de dados com maior poder informacional. As estatísticas dos testes Im, Pesaran e Shin, ADF-Fisher e PP-Fisher demonstraram não haver raízes unitárias nas séries não binárias, afastando o risco de regressões espúrias. Esses testes reforçam a robustez dos resultados empíricos.

Entre as limitações, há de se ressaltar que as conclusões são restritas aos 50 maiores bancos que compuseram a amostra. Entretanto, essas instituições respondem por 98,92\% dos ativos e por 99,04\% dos depósitos do sistema, na posição de dezembro de 2008, evidenciando a relevância dessas instituições no SFN.

Outra limitação é inerente ao tipo de estudo, denominado análise de conteúdo, no qual o resultado pode sofrer algum tipo de viés na construção da variável, em decorrência de suas características qualitativas. Como forma de mitigar esse risco, todas as análises de conteúdo realizadas por um pesquisador foram reavaliadas por outro, em processo de rodízio, como forma de dar uniformidade à análise.

Por fim, sugere-se a realização de futuras pesquisas sobre o mesmo tema, com diferentes abordagens metodológicas e que ampliem as análises apresentadas, com o uso, por exemplo, de variáveis instrumentais ou a aplicação de modelos de painel dinâmicos. 


\section{Referências}

ADMATI, A. R.; PFLEIDERER, P. Forcing firms to talk: financial disclosure regulation and externalities. Stanford: School of Business Stanford University, 1998.

ALVES, C. A. M. A divulgação do risco operacional segundo recomendações do Comitê de Basiléia: estudo em bancos com carteira comercial no Brasil. 2005. Dissertação (Mestrado em Administração) - Universidade Federal do Paraná.

; SALES, A. R. A divulgação do risco operacional com base na Resolução 3.380: um estudo dos dez maiores conglomerados bancários que atuam no Brasil. Revista Unieuro de Contabilidade (RUC), Brasília-DF, v. 1, n. 1, set. 2008.

BALTAGI, B. H. Econometric analysis of panel data. 4. ed. West Sussex, UK: John Wiley, 2008.

BANCO CENTRAL DO BRASIL (BCB). Circular 2.990, de 28.6.2000. Disponível em: $<$ http://www.bcb.gov.br>. Acesso em: 8 jun. 2009.

Comunicado 12.746, de 9 de dezembro de 2004. Disponível em:

<http://www.bcb.gov.br>. Acesso em: 5 abr. 2009.

Comunicado 16.137, de 27 de setembro de 2007. Disponível em:

<http://www.bcb.gov.br>. Acesso em: 5 abr. 2009.

Relatório dos 50 maiores bancos e o consolidado do sistema financeiro nacional.

Posição: Dezembro/2008. Disponível em: <http://www.bcb.gov.br/fis/TOP50>. Acesso em: 5 abr. 2009.

BASEL COMMITTEE ON BANKING SUPERVISION (BCBS). Enhancing Bank Transparency: public disclosure and supervisory information that promote safety and soundness in banking systems. Basel: BIS, 1998. Disponível em: <www.bis.org >. Acesso em: 4 jun. 2009.

Principles for the management of credit risk. Basel: BIS, 2000. Disponível em: <www.bis.org>. Acesso em: 4 jun. 2009.

Public disclosures by banks: results of the 2001 disclosure survey. Basel: BIS, 2003. Disponível em: <www.bis.org >. Acesso em: 4 jun. 2009.

International convergence on capital measurement and capital standards: a revised framework. Basel: BIS, 2004. Disponível em: <www.bis.org>. Acesso em: 4 jun. 2009.

History of the basel committee and its membership. Disponível em:

$<$ http://www.bis.org/bcbs/history.htm>. Acesso em: 5 abr. 2009.

BROOKS, C. Introductory econometrics for finance. Cambridge: Cambridge University Press, 2008. 
CARVALHO, D. B.; CALDAS, M. P. Basiléia II: abordagem prática para acompanhamento de risco operacional em instituições financeiras. Disponível em:

$<$ http://www.febraban.org.br/Arquivo/Servicos/Imprensa/Artigo_BasileiaII.pdf > . Acesso em: 12 maio 2009.

DANTAS, J. A. et. al. A dualidade entre os benefícios do disclosure e a relutância das organizações em aumentar o grau de evidenciação. Revista Economia e Gestão, PUC Minas, Belo Horizonte, v. 5, n. 11, p. 56-76, dez. 2005.

DI BENEDITO, E. A.; SILVA, R. N. Análise do nível de transparência das instituições financeiras no Brasil em Relação ao novo acordo de capitais (Basiléia II). ENANPAD, 31., 2007. Rio de Janeiro, Anais... Rio de Janeiro: ANPAD, 2007.

DOORNIK, B. F. N. V. et al. Transparência das demonstrações contábeis após o acordo de Basiléia 2: um estudo temporal dos 10 maiores bancos do país. ENANPAD, 31., 2007, Rio de Janeiro. Anais... Rio de Janeiro: ANPAD, 2007.

ENG, L. L.; MAK, Y. T. Corporate governance and voluntary disclosure. Journal of Accounting and Public Policy, v. 22, 2003.

FERREIRA, C.; ARAÚJO, E. Disclosure em instituições financeiras: uma análise comparativa entre Basiléia II e a prática brasileira. In: Semana de Contabilidade do Banco Central, 10., 2004, Brasília. Anais... Brasília: BCB, 2004, p. 73-92.

GOULART, A. M. C. Evidenciação Contábil do Risco de Mercado por Instituições Financeiras no Brasil. 2003. Dissertação (Mestrado em Controladoria e Contabilidade) Faculdade de Economia, Administração e Contabilidade, Universidade de São Paulo.

; CARVALHO, L. N. Evidenciação Contábil do Risco de Mercado por Instituições Financeiras no Brasil. Unb Contábil, Brasília, v. 7, n. 1, p. 9-32, 2004.

G-20 WORKING GROUP. Enhancing sound regulation and strengthening transparency: final report, Mar. 2009. Disponível em: <www.g20.org>. Acesso em: 4 jun. 2009.

IUDÍCIBUS, S. Teoria da contabilidade. 6. ed. São Paulo: Atlas, 2000.

MARQUES, L. D. Modelos dinâmicos com dados em painel: revisão de literatura. Centro de Estudos Macroeconomicos e Previsão - Faculdade de Economia do Porto, out. 2000. Disponível em: <http://fep.up.pt/investigacao/workingpapers>. Acesso em: 4 jun. 2009.

NIYAMA, J. K. Contribuição à avaliação do nível de qualidade da evidenciação contábil das empresas pertencentes ao Sistema Financeiro Habitacional - SFH. 1989. 310 p. Tese (Doutorado em Contabilidade) - Faculdade de Economia, Administração e Contabilidade da Universidade de São Paulo.

RONEN, J.; YAARI, V. Incentives for voluntary disclosure. Journal of Financial Markets, v. $5,2002$. 
SALOTTI, B. M.; YAMAMOTO, M. M. Divulgação voluntária da demonstração dos fluxos de caixa no mercado de capitais brasileiro. Revista Contabilidade e Finanças, São Paulo, v. 19, n. 48, p. 37-49, set./dez. 2008.

VERRECCHIA, R. E. Essays on disclosure. Journal of Accounting and Economics, n. 32, p. 97-180, 2001.

WATSON, A. et al. Voluntary disclosure of accounting ratios in the UK. British Accounting Review, v. 34, 2002.

XAVIER, P. Transparência das demonstrações contábeis dos bancos no Brasil: estudo de caso sob a perspectiva do Acordo "Basiléia 2". 2003. Dissertação (Mestrado em

Controladoria e Contabilidade) - Faculdade de Economia, Administração e Contabilidade, Universidade de São Paulo. 